\title{
ORBIFOLD ZETA FUNCTIONS FOR DUAL INVERTIBLE POLYNOMIALS
}

\author{
WOLFGANG EBELING ${ }^{1}$ AND SABIR M. GUSEIN-ZADE ${ }^{2}$ \\ ${ }^{1}$ Leibniz Universität Hannover, Institut für Algebraische Geometrie, Postfach 6009, \\ 30060 Hannover, Germany (ebeling@math.uni-hannover.de) \\ ${ }^{2}$ Moscow State University, Faculty of Mechanics and Mathematics, Moscow, \\ GSP-1, 119991, Russia (sabir@mccme.ru)
}

(Received 31 October 2014)

\begin{abstract}
An invertible polynomial in $n$ variables is a quasi-homogeneous polynomial consisting of $n$ monomials so that the weights of the variables and the quasi-degree are well defined. In the framework of the construction of mirror symmetric orbifold Landau-Ginzburg models, Berglund, Hübsch and Henningson considered a pair $(f, G)$ consisting of an invertible polynomial $f$ and an abelian group $G$ of its symmetries together with a dual pair $(\tilde{f}, \tilde{G})$. Here we study the reduced orbifold zeta functions of dual pairs $(f, G)$ and $(\tilde{f}, \tilde{G})$ and show that they either coincide or are inverse to each other depending on the number $n$ of variables.
\end{abstract}

Keywords: invertible polynomial; group action; monodromy; orbifold zeta function

2010 Mathematics subject classification: Primary 14J33; 14R20; 57R18; 58K10

\section{Introduction}

Berglund and Hübsch $[\mathbf{3}]$ proposed a method to construct some mirror symmetric pairs of manifolds. Their construction involves a polynomial $f$ of a special form, a so-called invertible one, and its Berglund-Hübsch transpose $\tilde{f}$. In [3] these polynomials appeared as potentials of Landau-Ginzburg models. This construction was generalized in $[\mathbf{2}]$ to orbifold Landau-Ginzburg models described by pairs $(f, G)$, where $f$ is an invertible polynomial and $G$ is a (finite) abelian group of symmetries of $f$. For a pair $(f, G)$ one defines the dual pair $(\tilde{f}, \tilde{G})$. Some symmetries between invariants of the pairs $(f, G)$ and $(\tilde{f}, \tilde{G})$ corresponding to the orbifolds defined by the equations $f=0$ and $\tilde{f}=0$ in weighted projective spaces were described in $[\mathbf{2 , 1 1}$. Some duality (symmetry) properties of the singularities defined by $f$ and $\tilde{f}$ were observed in $[\mathbf{5 - 7 , 1 4}]$. In particular, in $[\mathbf{5}$, Theorem 1] it was shown that the reduced orbifold Euler characteristics of the Milnor fibres of $f$ and $\tilde{f}$ with the actions of the groups $G$ and $\tilde{G}$, respectively, coincide up to sign.

Here we consider the (reduced) orbifold zeta function defined in [7, Definition 5.10]. One can say that it collects information about the eigenvalues of monodromy operators 
modified by so-called age (or fermion) shifts. We show that the (reduced) orbifold zeta functions of Berglund-Hübsch-Henningson dual pairs $(f, G)$ and $(\tilde{f}, \tilde{G})$ either coincide or are inverse to each other depending on the number $n$ of variables. This is a refinement of the above mentioned result of [5] that means that the degrees of these zeta functions coincide up to sign.

\section{Invertible polynomials}

A quasi-homogeneous polynomial $f$ in $n$ variables is called invertible (see [13]) if it contains $n$ monomials, i.e. it is of the form

$$
f\left(x_{1}, \ldots, x_{n}\right)=\sum_{i=1}^{n} a_{i} \prod_{j=1}^{n} x_{j}^{E_{i j}}
$$

for $a_{i} \in \mathbb{C}^{*}=\mathbb{C} \backslash\{0\}$, and the matrix $E=\left(E_{i j}\right)$ (with non-negative integer entries) is non-degenerate: $\operatorname{det} E \neq 0$. Without loss of generality we may assume that $a_{i}=1$ for $i=1, \ldots, n$ and that $\operatorname{det} E>0$.

The Berglund-Hübsch transpose $\tilde{f}$ of the invertible polynomial (2.1) is

$$
\tilde{f}\left(x_{1}, \ldots, x_{n}\right)=\sum_{i=1}^{n} a_{i} \prod_{j=1}^{n} x_{j}^{E_{j i}},
$$

i.e. it is defined by the transpose $E^{\mathrm{T}}$ of the matrix $E$.

The (diagonal) symmetry group of the invertible polynomial $f$ is the group $G_{f}$ of diagonal linear transformations of $\mathbb{C}^{n}$ preserving $f$ :

$$
G_{f}=\left\{\left(\lambda_{1}, \ldots, \lambda_{n}\right) \in\left(\mathbb{C}^{*}\right)^{n}: f\left(\lambda_{1} x_{1}, \ldots, \lambda_{n} x_{n}\right)=f\left(x_{1}, \ldots, x_{n}\right)\right\} .
$$

This group is finite and its order $\left|G_{f}\right|$ is equal to $d=\operatorname{det} E$ (see $[\mathbf{1 3},(10)$ and (18)] and $[\mathbf{6}$, Proposition 1]). The polynomial $f$ is quasi-homogeneous with respect to the rational weights $q_{1}, \ldots, q_{n}$ defined by the equation

$$
E\left(q_{1}, \ldots, q_{n}\right)^{\mathrm{T}}=(1, \ldots, 1)^{\mathrm{T}},
$$

i.e.

$$
f\left(\exp \left(2 \pi \mathrm{i} q_{1} \tau\right) x_{1}, \ldots, \exp \left(2 \pi \mathrm{i} q_{n} \tau\right) x_{n}\right)=\exp (2 \pi \mathrm{i} \tau) f\left(x_{1}, \ldots, x_{n}\right) .
$$

The Milnor fibre of the polynomial $f$ is the manifold

$$
V_{f}=\left\{\left(x_{1}, \ldots, x_{n}\right) \in \mathbb{C}^{n}: f\left(x_{1}, \ldots, x_{n}\right)=1\right\} .
$$

(The Milnor fibre defined in this way coincides with (i.e. is diffeomorphic to) the one given by the general definition below (see $\S 3$ ) since the polynomial $f$ is quasi-homogeneous.) The monodromy transformation of the polynomial $f$ (see below) is induced by the element

$$
g_{0}=\left(\exp \left(2 \pi \mathrm{i} q_{1}\right), \ldots, \exp \left(2 \pi \mathrm{i} q_{n}\right)\right) \in G_{f} .
$$

(In $[\mathbf{1 2}]$ the element $g_{0}$ is called the 'exponential grading operator'.) 
For a finite abelian group $G$, let $G^{*}=\operatorname{Hom}\left(G, \mathbb{C}^{*}\right)$ be its group of characters. (The groups $G$ and $G^{*}$ are isomorphic, but not in a canonical way. The group $\left(G^{*}\right)^{*}$ is canonically isomorphic to $G$.) One can show that the symmetry group $G_{\tilde{f}}$ of the BerglundHübsch transpose $\tilde{f}$ of an invertible polynomial $f$ is canonically isomorphic to $G_{f}^{*}$ (see, for example, $[\mathbf{6}])$. The duality between $G_{f}$ and $G_{\tilde{f}}$ is defined by the pairing

$$
\langle\underline{\lambda}, \underline{\mu}\rangle_{E}=\exp \left(2 \pi \mathrm{i}(\underline{\alpha}, \underline{\beta})_{E}\right)
$$

where

$$
\begin{gathered}
\underline{\lambda}=\left(\exp \left(2 \pi \mathrm{i} \alpha_{1}\right), \ldots, \exp \left(2 \pi \mathrm{i} \alpha_{n}\right)\right) \in G_{\tilde{f}}, \quad \underline{\mu}=\left(\exp \left(2 \pi \mathrm{i} \beta_{1}\right), \ldots, \exp \left(2 \pi \mathrm{i} \beta_{n}\right)\right) \in G_{f}, \\
\underline{\alpha}=\left(\alpha_{1}, \ldots, \alpha_{n}\right), \quad \underline{\beta}=\left(\beta_{1}, \ldots, \beta_{n}\right), \\
(\underline{\alpha}, \underline{\beta})_{E}:=\left(\alpha_{1}, \ldots, \alpha_{n}\right) E\left(\beta_{1}, \ldots, \beta_{n}\right)^{\mathrm{T}}
\end{gathered}
$$

(see [6, Proposition 2]).

Definition 2.1 (Berglund and Henningson [2]). For a subgroup $H \subset G_{f}$, its dual $\tilde{H} \subset G_{\tilde{f}}=G_{f}^{*}$ is the kernel of the natural map $i^{*}: G_{f}^{*} \rightarrow H^{*}$ induced by the inclusion $i: H \hookrightarrow G_{f}$.

One can see that $|H||\tilde{H}|=\left|G_{f}\right|=\left|G_{\tilde{f}}\right|$. The following result was proved in $[\mathbf{1 2}, \S 3.1]$.

Lemma 2.2. Let $G_{f, 0}=\left\langle g_{0}\right\rangle$ be the subgroup of $G_{f}$ generated by the monodromy transformation. One has $\widetilde{G_{f, 0}}=G_{\tilde{f}} \cap \operatorname{SL}(n, \mathbb{C})$.

Proof. For $\underline{\lambda}=\left(\lambda_{1}, \ldots, \lambda_{n}\right)=\left(\exp \left(2 \pi \mathrm{i} \alpha_{1}\right), \ldots, \exp \left(2 \pi \mathrm{i} \alpha_{n}\right)\right) \in G_{\tilde{f}},\left\langle\underline{\lambda}, g_{0}\right\rangle_{E}=1$ if and only if

$$
\left(\alpha_{1}, \ldots, \alpha_{n}\right) E\left(q_{1}, \ldots, q_{n}\right)^{\mathrm{T}} \in \mathbb{Z}
$$

One has $E\left(q_{1}, \ldots, q_{n}\right)^{\mathrm{T}}=(1, \ldots, 1)^{\mathrm{T}}$. Therefore, $\left(\alpha_{1}, \ldots, \alpha_{n}\right)(1, \ldots, 1)^{\mathrm{T}} \in \mathbb{Z}$, i.e. $\sum_{i} \alpha_{i} \in$ $\mathbb{Z}, \prod_{i} \lambda_{i}=1$. This means that $\underline{\lambda} \in \operatorname{SL}(n, \mathbb{C})$.

\section{Orbifold zeta function}

The zeta function $\zeta_{h}(t)$ of a (proper, continuous) transformation $h: X \rightarrow X$ of a topological space $X$ is the rational function defined by

$$
\zeta_{h}(t)=\prod_{q \geqslant 0}\left(\operatorname{det}\left(\operatorname{id}-t h^{*} \mid H_{c}^{q}(X ; \mathbb{R})\right)\right)^{(-1)^{q}},
$$

where $H_{\mathrm{c}}^{q}(X ; \mathbb{R})$ denotes the cohomology with compact support. The degree of the zeta function $\zeta_{h}(t)$, i.e. the degree of the numerator minus the degree of the denominator, is equal to the Euler characteristic $\chi(X)$ of the space $X$ (defined via cohomology with compact support).

Remark 3.1. If a transformation $h: X \rightarrow X$ defines on $X$ a free action of the cyclic group of order $m$ (i.e. if $h^{m}(x)=x, h^{k}(x) \neq x$ for $0<k<m, x \in X$ ), then $\zeta_{h}(t)=\left(1-t^{m}\right)^{\chi(X) / m}$. 
Let $f:\left(\mathbb{C}^{n}, 0\right) \rightarrow(\mathbb{C}, 0)$ be a germ of a holomorphic function. The Milnor fibre $V_{f}$ of the germ $f$ is the manifold $\{f=\varepsilon\} \cap B_{\delta}^{2 n}$, where $B_{\delta}^{2 n}$ is the ball of radius $\delta$ centred at the origin in $\mathbb{C}^{n}, 0<|\varepsilon| \ll \delta, \delta$ is small enough. The monodromy zeta function, i.e. the zeta function of the monodromy transformation $h_{f}$ of the germ $f$ (and also of its restriction to a subspace), is of the form $\prod_{m \geqslant 1}\left(1-t^{m}\right)^{s_{m}}$, where $s_{m}$ are integers such that only finitely many of them are different from zero (see, for example, [1, Théorème 3]). In particular, all roots and/or poles of the monodromy zeta function are roots of unity.

The orbifold (monodromy) zeta function was essentially defined in [7, Definition 5.10]. It is related to the Poincaré polynomial for the Ramond ground states of [9].

Let $G$ be a finite group acting linearly on the space $\mathbb{C}^{n}$ and let the germ $f:\left(\mathbb{C}^{n}, 0\right) \rightarrow$ $(\mathbb{C}, 0)$ be $G$-invariant. One may assume that the monodromy transformation $h_{f}$ of the germ $f$ is $G$-invariant. For an element $g \in G$, its age $[\mathbf{1 0}, \S 2.1]$ (or fermion shift number $[\mathbf{1 6},(3.17)])$ is defined by age $(g):=\sum_{i=1}^{n} \alpha_{i}$, where in a certain basis in $\mathbb{C}^{n}$ one has

$$
g=\operatorname{diag}\left(\exp \left(2 \pi \mathrm{i} \alpha_{1}\right), \ldots, \exp \left(2 \pi \mathrm{i} \alpha_{n}\right)\right)
$$

with $0 \leqslant \alpha_{i}<1$.

Remark 3.2. The map $\exp (2 \pi \mathrm{i}$ age $(\cdot)): G \rightarrow \mathbb{C}^{*}$ is a group homomorphism. It coincides with the representation of $G$ induced on the $n$th exterior power of the space $\mathbb{C}^{n}$. If $f$ is an invertible polynomial and $G$ is the group $G_{f}$ of its symmetries, then it is an element of $G_{f}^{*}=G_{\tilde{f}}$.

For a rational function $\varphi(t)$ of the form $\prod_{i}\left(1-\alpha_{i} t\right)^{r_{i}}$ with only finitely many of the exponents $r_{i} \in \mathbb{Z}$ different from zero, its $g$-age shift is defined by

$$
(\varphi(t))_{g}=\prod_{i}\left(1-\alpha_{i} \exp (-2 \pi \mathrm{i} \operatorname{age}(g)) t\right)^{r_{i}}
$$

i.e. all its roots and/or poles are multiplied by $\exp (2 \pi \mathrm{i}$ age $(g)) \in \mathbb{C}^{*}$.

Let Conj $G$ be the set of conjugacy classes of elements of $G$. For a class $[g] \in \operatorname{Conj} G$, let $g \in G$ be a representative of it. Let $C_{G}(g)=\left\{h \in G: h^{-1} g h=g\right\}$ be the centralizer of the element $g$ in $G$. Let $\left(\mathbb{C}^{n}\right)^{g}$ be the fixed-point set of the element $g$, let $V_{f}^{g}=$ $V_{f} \cap\left(\mathbb{C}^{n}\right)^{g}$ be the corresponding part of the Milnor fibre, and let $\hat{V}_{f}^{g}=V_{f}^{g} / C_{G}(g)$ be the corresponding quotient space (the 'twisted sector' in terms of [4]). One may assume that the monodromy transformation preserves $V_{f}^{g}$ for each $g$. Let $\hat{h}_{f}^{g}: \hat{V}_{f}^{g} \rightarrow \hat{V}_{f}^{g}$ be the corresponding map (monodromy) on the quotient space. Its zeta function $\zeta_{\hat{h}_{f}^{g}}(t)$ depends only on the conjugacy class of $g$.

Definition 3.3. The orbifold zeta function of the pair $(f, G)$ is defined by

$$
\zeta_{f, G}^{\mathrm{orb}}(t)=\prod_{[g] \in \operatorname{Conj} G}\left(\zeta_{\hat{h}_{f}^{g}}(t)\right)_{g} .
$$

One can see that the degree of $\zeta_{f, G}^{\text {orb }}(t)$ is equal to the orbifold Euler characteristic of $\left(V_{f}, G\right)$ (see, for example, $[\mathbf{5}, \mathbf{8}]$ ).

For an abelian $G, \hat{V}_{f}^{g}=V_{f}^{g} / G$ and the product in (3.2) runs over all elements $g \in G$. 
Definition 3.4. The reduced orbifold zeta function $\bar{\zeta}_{f, G}^{\mathrm{orb}}(t)$ is defined by

$$
\bar{\zeta}_{f, G}^{\mathrm{orb}}(t)=\zeta_{f, G}^{\mathrm{orb}}(t) / \prod_{[g] \in \operatorname{Conj} G}(1-t)_{g}
$$

(cf. $(3.2)$ ).

Now let $G$ be abelian. One can assume that the action of $G$ on $\mathbb{C}^{n}$ is diagonal, and therefore it respects the decomposition of $\mathbb{C}^{n}$ into the coordinate tori. For a subset $I \subset I_{0}=\{1,2, \ldots, n\}$, let

$$
\left(\mathbb{C}^{*}\right)^{I}:=\left\{\left(x_{1}, \ldots, x_{n}\right) \in \mathbb{C}^{n}: x_{i} \neq 0 \text { for } i \in I, x_{i}=0 \text { for } i \notin I\right\}
$$

be the corresponding coordinate torus. Let $V_{f}^{I}=V_{f} \cap\left(\mathbb{C}^{*}\right)^{I}$. One has $V_{f}=\coprod_{I \subset I_{0}} V_{f}^{I}$. Since the action of $G$ is diagonal, $G$ preserves this decomposition, and thus acts on each subspace $V_{f}^{I}$. Let $G^{I} \subset G$ be the isotropy subgroup of the action of $G$ on the torus $\left(\mathbb{C}^{*}\right)^{I}$. (All points $x$ of the torus $\left(\mathbb{C}^{*}\right)^{I}$ have one and the same isotropy subgroup $G^{I}:=G_{x}=\{g \in G: g x=x\}$. One has $G^{I}=G \cap G_{f}^{I}$.) The monodromy transformation $h_{f}$ is assumed to be $G$-invariant and to respect the decomposition of the Milnor fibre $V_{f}$ into the parts $V_{f}^{I}$. Let $h_{f}^{I}$ and $\hat{h}_{f}^{I}$ be the corresponding (monodromy) transformations of $V_{f}^{I}$ and $V_{f}^{I} / G$, respectively. One can define in the same way as above the orbifold zeta function corresponding to the part $V_{f}^{I}$ of the Milnor fibre:

$$
\zeta_{f, G}^{\mathrm{orb}, I}(t)=\prod_{g \in G}\left(\zeta_{\hat{h}_{f}^{I, g}}(t)\right)_{g} .
$$

One has

$$
\zeta_{f, G}^{\mathrm{orb}}(t)=\prod_{I \subset I_{0}} \zeta_{f, G}^{\mathrm{orb}, I}(t)
$$

This follows from the multiplicativity of the (usual) zeta function with respect to a partition of the space germ. (Here and below one may assume that $I$ is not empty since for $I=\emptyset$ the corresponding factor is trivial.) Since the isotropy subgroups of all points of $\left(\mathbb{C}^{*}\right)^{I}$ are the same (equal to $G^{I}$ ), the equation (3.3) reduces to

$$
\zeta_{f, G}^{\mathrm{orb}, I}(t)=\prod_{g \in G^{I}}\left(\zeta_{\hat{h}_{f}^{I}}(t)\right)_{g} .
$$

The (monodromy) zeta function $\zeta_{\hat{h}_{f}^{I}}(t)$ has the form $\prod_{m \geqslant 0}\left(1-t^{m}\right)^{s_{m}}$ with only a finite number of the exponents $s_{m}$ different from zero. Let us compute $\prod_{g \in G^{I}}\left(1-t^{m}\right)_{g}$.

Lemma 3.5. One has

$$
\prod_{g \in G^{I}}\left(1-t^{m}\right)_{g}=\left(1-t^{\operatorname{lcm}(m, k)}\right)^{m\left|G^{I}\right| / \operatorname{lcm}(m, k)},
$$

where $k=\left|G^{I} / G^{I} \cap \mathrm{SL}(n, \mathbb{C})\right|, \operatorname{lcm}(\cdot, \cdot)$ denotes the least common multiple. 
Proof. The roots of the binomial $\left(1-t^{m}\right)$ are all the $m$ th roots of unity. The map $\exp (2 \pi$ i age $(\cdot)): G^{I} \rightarrow \mathbb{C}^{*}$ is a group homomorphism. Its kernel coincides with $G^{I} \cap$ $\mathrm{SL}(n, \mathbb{C})$. Therefore, its image consists of all the $k$ th roots of unity (each one corresponds to $\left|G^{I} \cap \mathrm{SL}(n, \mathbb{C})\right|$ elements of $\left.G^{I}\right)$. Thus, the roots of $\prod_{g \in G^{I}}\left(1-t^{m}\right)_{g}$ are all the roots of unity of degree $\operatorname{lcm}(m, k)$ with equal multiplicities. This means that $\prod_{g \in G^{I}}\left(1-t^{m}\right)_{g}=$ $\left(1-t^{\operatorname{lcm}(m, k)}\right)^{s}$. The exponent $s$ is determined by the number of roots.

\section{Orbifold zeta functions for invertible polynomials}

Let $(f, G)$ be a pair consisting of an invertible polynomial $f$ in $n$ variables and a group $G \subset G_{f}$ of its (diagonal) symmetries and let $(\tilde{f}, \tilde{G})$ be the Berglund-HübschHenningson dual pair $\left(\tilde{G} \subset G_{\tilde{f}}\right)$. (We do not assume that the invertible polynomials are non-degenerate, i.e. that they have isolated critical points at the origin.)

Theorem 4.1. One has

$$
\bar{\zeta}_{\tilde{f}, \tilde{G}}^{\mathrm{orb}}(t)=\left(\bar{\zeta}_{f, G}^{\mathrm{orb}}(t)\right)^{(-1)^{n}} .
$$

Proof. We use the notation from $\S 3$. One has

$$
\bar{\zeta}_{f, G}^{\mathrm{orb}}(t)=\prod_{I \subset I_{0}} \zeta_{f, G}^{\mathrm{orb}, I}(t) / \prod_{g \in G}(1-t)_{g} .
$$

Let $\mathbb{Z}^{n}$ be the lattice of monomials in the variables $x_{1}, \ldots, x_{n}\left(\right.$ an $n$-tuple $\left(k_{1}, \ldots, k_{n}\right) \in$ $\mathbb{Z}^{n}$ corresponds to the monomial $\left.x_{1}^{k_{1}} \cdots x_{n}^{k_{n}}\right)$ and let $\mathbb{Z}^{I}:=\left\{\left(k_{1}, \ldots, k_{n}\right) \in \mathbb{Z}^{n}: k_{i}=\right.$ 0 for $i \notin I\}$. For a polynomial $F$ in the variables $x_{1}, \ldots, x_{n}$, let $\operatorname{supp} F \subset \mathbb{Z}^{n}$ be the set of monomials (with non-zero coefficients) in $F$.

The elements of the subgroup $G_{f, 0} \cap G_{f}^{I}$ act on $V_{f}^{I}$ trivially. The monodromy transformation defines a free action of the cyclic group $G_{f, 0} /\left(G_{f, 0} \cap G_{f}^{I}\right)$ on $V_{f}^{I}$. Therefore, the monodromy transformation on $V_{f}^{I} / G$ defines an action of the cyclic group $G_{f, 0} /\left(G_{f, 0} \cap\left(G+G_{f}^{I}\right)\right)$, which is also free. According to Remark 3.1, the zeta function is given by

$$
\zeta_{\hat{h}_{f}^{I}}(t)=\left(1-t^{m_{I}}\right)^{s_{I}},
$$

where

$$
m_{I}=\left|G_{f, 0} /\left(G_{f, 0} \cap\left(G+G_{f}^{I}\right)\right)\right|=\frac{\left|G+G_{f}^{I}+G_{f, 0}\right|}{\left|G+G_{f}^{I}\right|},
$$

$s_{I}=\chi\left(V_{f}^{I} / G\right) / m_{I}=\chi\left(V_{f}^{I}\right) /\left(m_{I}\left|G / G \cap G_{f}^{I}\right|\right)$.

Let $I$ be a proper subset of $I_{0}=\{1, \ldots, n\}$ (i.e. $I \neq \emptyset, I \neq I_{0}$ ), and let $\bar{I}=I_{0} \backslash I$. If $(\operatorname{supp} f) \cap \mathbb{Z}^{I}$ consists of fewer than $|I|$ points, i.e. if $f$ has fewer than $|I|$ monomials in the variables $x_{i}$ with $i \in I$, then $\chi\left(V_{f}^{I}\right)=0$ (for example, due to the Varchenko formula [15]) and therefore $\zeta_{\hat{h}_{f}^{I}}(t)=1, \zeta_{f, G}^{\text {orb }, I}(t)=1$. In this case $(\operatorname{supp} \tilde{f}) \cap \mathbb{Z}^{\bar{I}}$ consists of fewer than $|\bar{I}|$ points and therefore we have $\zeta_{\tilde{f}, \tilde{G}}^{\text {orb }, I}(t)=1$.

Let $\left|(\operatorname{supp} f) \cap \mathbb{Z}^{I}\right|=|I|$. From (4.3) and Lemma 3.5 it follows that

$$
\zeta_{f, G}^{\mathrm{orb}, I}(t)=\left(1-t^{\mathrm{lcm}\left(m_{I}, k_{I}\right)}\right)^{s_{I}^{\prime}},
$$


where

$$
k_{I}=\frac{\left|G \cap G_{f}^{I}\right|}{\left|G \cap G_{f}^{I} \cap \operatorname{SL}(n, \mathbb{C})\right|}
$$

and $s_{I}^{\prime}$ is some integer. (Namely, $s_{I}^{\prime}=\left(\chi\left(V_{f}^{I} / G\right) \cdot\left|G^{I}\right|\right) / \operatorname{lcm}\left(m_{I}, k_{I}\right)$. We shall not use this equation explicitly.) Therefore,

$$
\zeta_{f, G}^{\mathrm{orb}, I}(t)=\left(1-t^{\ell_{I}}\right)^{s_{I}^{\prime}},
$$

where

$$
\ell_{I}=\operatorname{lcm}\left(\frac{\left|G+G_{f}^{I}+G_{f, 0}\right|}{\left|G+G_{f}^{I}\right|}, \frac{\left|G \cap G_{f}^{I}\right|}{\left|G \cap G_{f}^{I} \cap \operatorname{SL}(n, \mathbb{C})\right|}\right) .
$$

In this case $\left|(\operatorname{supp} \tilde{f}) \cap \mathbb{Z}^{\bar{I}}\right|=|\bar{I}|$, and therefore

$$
\zeta_{\tilde{f}, \tilde{G}}^{\operatorname{orb}, \bar{I}}(t)=\left(1-t^{\tilde{\ell}_{\bar{I}}}\right)^{\tilde{s}_{\bar{I}}^{\prime}},
$$

where

$$
\tilde{\ell}_{\bar{I}}=\operatorname{lcm}\left(\frac{\left|\tilde{G}+G_{\tilde{f}}^{\bar{I}}+G_{\tilde{f}, 0}\right|}{\left|\tilde{G}+G_{\tilde{f}}^{\bar{I}}\right|}, \frac{\left|\tilde{G} \cap G_{\tilde{f}}^{\bar{I}}\right|}{\left|\tilde{G} \cap G_{\tilde{f}}^{\bar{I}} \cap \operatorname{SL}(n, \mathbb{C})\right|}\right)
$$

and $\tilde{s}_{\bar{I}}^{\prime}$ is an integer. According to [6, Lemma 1], one has $G_{\tilde{f}}^{\bar{I}}=\widetilde{G_{f}^{I}}$; by Lemma 2.2, one has $\widetilde{G_{\tilde{f}, 0}}=G_{f} \cap \mathrm{SL}(n, \mathbb{C})$ and $\widetilde{G_{f, 0}}=G_{\tilde{f}} \cap \operatorname{SL}(n, \mathbb{C})$. This means that the subgroup $G+G_{f}^{I}+G_{f, 0} \subset G_{f}$ is dual to $\tilde{G} \cap G_{\tilde{f}}^{\bar{I}} \cap \operatorname{SL}(n, \mathbb{C}) \subset G_{\tilde{f}}$ and the subgroup $G+G_{f}^{I} \subset G_{f}$ is dual to $\tilde{G} \cap G_{\tilde{f}}^{\bar{I}} \subset G_{\tilde{f}}$. Therefore,

$$
\frac{\left|G+G_{f}^{I}+G_{f, 0}\right|}{\left|G+G_{f}^{I}\right|}=\frac{\left|\tilde{G} \cap G_{\tilde{f}}^{\bar{I}}\right|}{\left|\tilde{G} \cap G_{\tilde{f}}^{\bar{I}} \cap \operatorname{SL}(n, \mathbb{C})\right|} .
$$

In the same way

$$
\frac{\left|G \cap G_{f}^{I}\right|}{\left|G \cap G_{f}^{I} \cap \operatorname{SL}(n, \mathbb{C})\right|}=\frac{\left|\tilde{G}+G_{\tilde{f}}^{\bar{I}}+G_{\tilde{f}, 0}\right|}{\left|\tilde{G}+G_{\tilde{f}}^{\bar{I}}\right|},
$$

and therefore $\ell_{I}=\widetilde{\ell}_{\bar{I}}$. In [5] it was shown that $\ell_{I} s_{I}^{\prime}=(-1)^{n} \widetilde{\ell}_{\bar{I}} \tilde{s}_{\bar{I}}^{\prime}$. Thus, $s_{I_{\bar{I}}}^{\prime}=(-1)^{n} \tilde{s}_{\bar{I}}^{\prime}$. Therefore, the factor $\zeta_{f, G}^{\text {orb, } I}(t)$ in $(4.2)$ for $\bar{\zeta}_{f, G}^{\text {orb }}(t)$ is equal to the factor $\left(\zeta_{\tilde{f}, \tilde{G}}^{\text {orb }, \bar{I}}(t)\right)^{(-1)^{n}}$ in the corresponding equation for $\left(\bar{\zeta}_{\tilde{f}, \tilde{G}}^{\text {orb }}(t)\right)^{(-1)^{n}}$.

Now let $I=I_{0}$. One has $G_{f}^{I_{0}}=\{0\}$ and therefore $\zeta_{f, G}^{\text {orb }, I_{0}}(t)=\zeta_{\hat{h}_{f}^{I_{0}}}(t)=\left(1-t^{m_{I_{0}}}\right)^{s_{I_{0}}}$, where $m_{I_{0}}=\left|G_{f, 0} / G \cap G_{f, 0}\right|=\left|G+G_{f, 0}\right| /|G|$. On the other hand, by Lemma 3.5, one has

$$
\prod_{g \in \tilde{G}}(1-t)_{g}=\left(1-t^{\tilde{k}}\right)^{\tilde{r}}
$$

where

$$
\tilde{k}=\frac{|\tilde{G}|}{|\tilde{G} \cap \operatorname{SL}(n, \mathbb{C})|}
$$

and $\tilde{r}$ is some integer. Due to Lemma 2.2, the subgroup $\tilde{G} \cap \operatorname{SL}(n, \mathbb{C}) \subset G_{\tilde{f}}$ is dual to the subgroup $G+G_{f, 0} \subset G_{f}$. Therefore, $m_{I_{0}}=\tilde{k}$. In [5] it was shown that $m_{I_{0}} s_{I_{0}}=$ 
$(-1)^{n-1} \tilde{k} \tilde{r}$. Therefore, $s_{I_{0}}=(-1)^{n-1} \tilde{r}$ and the factor $\zeta_{f, G}^{\text {orb }, I_{0}}(t)$ in $(4.2)$ for $\bar{\zeta}_{f, G}^{\text {orb }}(t)$ is equal to the factor $\left(\prod_{g \in \tilde{G}}(1-t)_{g}\right)^{(-1)^{n-1}}$ in the corresponding equation for $\left(\bar{\zeta}_{\tilde{f}, \tilde{G}}^{\mathrm{orb}}(t)\right)^{(-1)^{n}}$.

Remark 4.2. Informally one can say that, in (4.6) for the exponent $\ell_{I}$, the first argument of the least common multiple is connected with the monodromy action and the second one with the age shift. (This means that in the computation of the orbifold zeta function according to Definition 3.3 the first argument originates from the (usual) monodromy zeta function $\zeta_{\hat{h}_{f}^{g}}(t)$, whereas the second one originates from the age shifts.) The duality interchanges these numbers. The one for the pair $(f, G)$ connected with the monodromy action is equal to the one for the dual pair $(\tilde{f}, \tilde{G})$ connected with the age shift and vice versa (see (4.7)).

Acknowledgements. This work was partly supported by DFG (Mercator fellowship, Grant Eb 102/8-1), Grants RFBR-16-01-00409 and NSh-2789.2016.1.

\section{References}

1. N. A'CAmpo, La fonction zêta d'une monodromie, Comment. Math. Helv. 50 (1975), 233-248.

2. P. Berglund and M. Henningson, Landau-Ginzburg orbifolds, mirror symmetry and the elliptic genus, Nucl. Phys. B 433 (1995), 311-332.

3. P. Berglund And T. HüBsch, A generalized construction of mirror manifolds, Nucl. Phys. B 393 (1993), 377-391.

4. W. Chen And Y. Ruan, A new cohomology theory of orbifold, Commun. Math. Phys. 248(1) (2004), 1-31.

5. W. Ebeling and S. M. Gusein-Zade, Orbifold Euler characteristics for dual invertible polynomials, Mosc. Math. J. 12(1) (2012), 49-54.

6. W. Ebeling And S. M. Gusein-Zade, Saito duality between Burnside rings for invertible polynomials, Bull. Lond. Math. Soc. 44(4) (2012), 814-822.

7. W. Ebeling And A. TAKahashi, Mirror symmetry between orbifold curves and cusp singularities with group action, Int. Math. Res. Not. 2013 (2013), 2240-2270.

8. F. Hirzebruch And Th. Höfer, On the Euler number of an orbifold, Math. Annalen 286 (1990), 255-260.

9. K. Intriligator And C. VAfa, Landau-Ginzburg orbifolds, Nucl. Phys. B 339 (1990), 95-120.

10. Y. Ito And M. ReID, The McKay correspondence for finite subgroups of SL(3, $\mathbb{C})$, in Higher-dimensional complex varieties, De Gruyter Expositions in Mathematics, pp. 221240 (De Gruyter, Berlin, 1996).

11. T. Kawai And S.-K. Yang, Duality of orbifoldized elliptic genera, Prog. Theor. Phys. Suppl. 118 (1995), 277-297.

12. M. KRAWITZ, FJRW-rings and Landau-Ginzburg mirror symmetry, Preprint (arXiv:0906 $.0796 ; 2009)$.

13. M. Kreuzer, The mirror map for invertible LG models, Phys. Lett. B 328(3-4) (1994), $312-318$.

14. A. TAKahashi, K. Saito's duality for regular weight systems and duality for orbifoldized Poincaré polynomials, Commun. Math. Phys. 205 (1999), 571-586.

15. A. N. VARChenko, Zeta-function of monodromy and Newton's diagram, Invent. Math. 37(3) (1976), 253-262.

16. E. ZAslow, Topological orbifold models and quantum cohomology rings, Commun. Math. Phys. 156(2) (1993), 301-331. 